\title{
Dectin-1-Syk-CARD9 Signaling Pathway in TB Immunity
}

\author{
Matthew Wagener', J. Claire Hoving ${ }^{1}$, Hlumani Ndlovu² and Mohlopheni J. Marakalala ${ }^{1 \star}$ \\ ' Division of Immunology, Department of Pathology, Institute of Infectious Disease and Molecular Medicine, University of \\ Cape Town, Cape Town, South Africa, ${ }^{2}$ Department of Integrative Biomedical Sciences, Faculty of Health Sciences, \\ University of Cape Town, Cape Town, South Africa
}

\section{OPEN ACCESS}

Edited by:

Roland Lang,

Universitätsklinikum Erlangen

Germany

Reviewed by:

Anca Dorhoi,

Friedrich Loeffler Institute

Greifswald, Germany

Ludovic Tailleux,

Institut Pasteur, France

*Correspondence:

Mohlopheni J. Marakalala

mj.marakalala@uct.ac.za

Specialty section:

This article was submitted to Molecular Innate Immunity,

a section of the journal

Frontiers in Immunology

Received: 21 November 2017

Accepted: 26 January 2018

Published: 13 February 2018

Citation:

Wagener M, Hoving JC, Ndlovu H and Marakalala MJ (2018)

Dectin-1-Syk-CARD9 Signaling

Pathway in TB Immunity.

Front. Immunol. 9:225.

doi: 10.3389/fimmu.2018.00225
One of the first steps toward mounting an effective immune response to Mycobacterium tuberculosis (Mtb) is recognition of the pathogen through pattern-recognition receptors (PRRs) expressed by innate immune cells. Activation of the PRR Dectin-1 by an unknown mycobacterial ligand triggers an intracellular signaling cascade involving numerous proteins, including spleen tyrosine kinase, protein kinase C-delta, and caspase recruitment domain family member 9 , some of which have been shown to influence host immune response to TB infection. Here, we review the role of Dectin-1 signaling pathway in anti-mycobacterial immunity and discuss its contribution in the control of Mtb infection, and potential applications in TB vaccine adjuvanticity.

Keywords: tuberculosis, Mycobacterium tuberculosis, dectin-1, C-type lectin receptors, innate immunity, TB immunity, pattern-recognition receptors, pathogen-associated molecular patterns

\section{INTRODUCTION}

A critical function of the innate immune system is differentiating between cells or components which are "self", and those of pathogenic microorganisms (1). Innate immune cells express patternrecognition receptors (PRRs) which recognize evolutionarily conserved microbial molecules, known as pathogen-associated molecular patterns (PAMPs) (2). Recognition via PRRs such as C-type lectin receptors (CLRs), NOD-like receptors, and toll-like receptors (TLRs) enable these cells to initiate responses to a broad range of potential pathogens.

When an innate immune cell, such as an alveolar macrophage, recognizes Mycobacterium tuberculosis (Mtb), a unique complement of PRRs will be activated. This triggers a signaling cascade within the innate cell, which results in the expression of immune modulators tailored to that pathogen. These immune modulators may trigger local inflammatory responses and provide the costimulation required for the activation and proliferation of adaptive immune cells, such as $\mathrm{CD} 4^{+} \mathrm{T}$ cells (2).

Understanding the role that PRR signaling plays in immunity to mycobacteria has been a recent research focus. Recent studies have shown that some receptors that activate the spleen tyrosine kinase (Syk)/caspase recruitment domain family member 9 (CARD9) signaling pathway may contribute to anti-mycobacterial defense. Several CLRs that recognize mycobacteria are known to utilize this pathway, including Dectin-1, Dectin-2, Mincle, and Clecsf8 $(3,4)$. These receptors are briefly discussed below, and the rest of the review will discuss the importance of Dectin-1-Syk-CARD9 signaling in orchestrating anti-mycobacterial immunity.

\section{Syk/CARD9-COUPLED CLRs IN TB IMMUNITY}

Dectin-2 recognizes mycobacterial mannosylated lipoarabinomannan (ManLAM) (5). This interaction results in the recruitment of immunoreceptor tyrosine-based activation motif (ITAM)-linked FcR $\gamma$, which links to Syk and CARD9, resulting in a cascade of downstream signaling and cellular 
activation $(5,6)$. Dectin-2 also induces production of anti- and pro-inflammatory cytokines IL-2, TNF, MIP-2, IL-6, and IL-10, in DCs stimulated with ManLAM and BCG. The interaction of this CLR with ManLAM has also been shown to induce T-cell responses (5). Dectin-2 deficiency results in increased pathological damage in mice infected with M. avium (5). Although Dectin-2 has been shown to recognize pathogenic Mtb strain, H37Rv, the in vivo protective role of this receptor against this strain is yet to be demonstrated.

Mincle interacts with mycobacteria via trehalose 6,6' dimycolate (TDM) $(7,8)$, the most abundant glycolipid on the cell wall of the bacilli (9). Like Dectin-2, this receptor is also coupled to the adaptor molecule, $\mathrm{FcR} \gamma$, which initiates Syk-mediated cellular responses $(9,10)$. Mincle has been shown to trigger pro-inflammatory cytokine production and nitric oxide (NO) in macrophages stimulated with TDM or its synthetic analog, trehalose 6,6-dibehenate (TDB). TDB also induces Mincle-driven adaptive Th1 and Th17 responses when used as an adjuvant to subunit vaccines in mice $(7,8,11)$. Despite these contributions to protective responses, Mincle has been shown to be dispensable for the control of Mtb infection in vivo (12), although contradicting results have been reported $(3,4,9)$.

Clecsf8 (MCL) is another FcR $\gamma$-coupled receptor that recognizes mycobacterial TDM (13). This CLR is known to positively regulate the expression of Mincle through a protein-protein complex interaction (14). Clecsf8-mediated cellular responses, which are dependent on the Syk/CARD9 complex, include phagocytosis, pro-inflammatory cytokine production, DC maturation, T-cell priming, and respiratory burst (14-16). Clecsf8-deficient mice are more susceptible to Mtb infection with increased lung bacillary loads, enhanced pathological damage with excessive neutrophilic infiltration, and early mortality (17). Clecsf8 polymorphisms are associated with TB susceptibility in humans (17).

Other Syk-coupled CLRs have been reported to recognize mycobacterial ligands. These have been reviewed elsewhere $(4,18)$, and they include DCAR, which recognizes glycolipds called PIMs (19), and SIGNR3, a DC-SIGN mouse homolog that recognizes ManLAM (20).

\section{DECTIN-1 STRUCTURE AND FUNCTION}

Dectin-1 was initially identified by subtractive cDNA cloning, using mRNA extracted from murine DCs (21). This PRR is expressed on various myeloid cells, including macrophages and other mononuclear cells, as well as a subpopulation of T cells (22). Consistent with its role in pathogen surveillance (23), Dectin-1 is highly expressed by immune cells residing in the mucosa of the lung (22) and gut (24). Dectin-1 is a glycosylated transmembrane receptor (type II) composed of two functional domains (see Figure 1). An extracellular C-type lectin domain (CTLD) binds $\beta$-glucans, polysaccharides that occur as $(1 \rightarrow 3)-\beta$-Dlinked glucose polymers, mainly found on the surface of fungi, plants, and some bacteria (25). Dectin-1 may also bind ligands other than $\beta$-glucans (21), as evidenced by the observation that mycobacteria, which do not contain $\beta$-glucans on their cell wall, seem to interact with Dectin-1 through a yet to be identified ligand (26). The second functional domain is found on the intracellular tail region, which houses an ITAM-like motif, called hemITAM (23).

\section{DECTIN-1 SIGNALING AND IMMUNE RESPONSES}

Dectin-1 initiates intracellular signaling via its hemITAM motif (Figure 2). Following ligand engagement by the CTLD, Src kinases mediate the tyrosine phosphorylation of the hemITAM domain (23), creating a docking site for Syk, which initiates a series of intracellular signaling cascades resulting in activation of the transcription factor nuclear factor $\kappa \mathrm{B}(\mathrm{NF}-\kappa \mathrm{B})(23,27,28)$. Dectin-1 can also drive non-canonical activation of NF- $\kappa \mathrm{B}$ by utilizing the serine-threonine kinase Raf-1 (29).

By influencing gene expression, Dectin-1 activation can lead to numerous downstream cellular responses, including expression of cytokines such as TNF- $\alpha$, IL-2, IL-10 and IL-12, and CXCL2 $(28,30,31)$. Dectin-1-mediated activation can also induce phagocytosis (32) and respiratory burst (33). Furthermore, Dectin-1 signaling has been shown to orchestrate adaptive immunity. DCs activated by Dectin-1 agonists are capable of differentiating naïve $\mathrm{CD}^{+} \mathrm{T}$ cells to a T helper- (Th-) 1 or Th17 phenotype both in vitro and in vivo (34). Dectin-1-activated DCs can also induce the maturation and proliferation of $\mathrm{CD}^{+} \mathrm{T}$ cells in vitro: the Dectin-1 agonist curdlan was found to act as an adjuvant for cytotoxic T lymphocyte cross-priming in vivo, which elicited potent responses capable of protecting mice from experimental tumor challenges (35). Thus, Dectin-1 signaling serves as a link that generates appropriate adaptive responses following immune recognition (36).

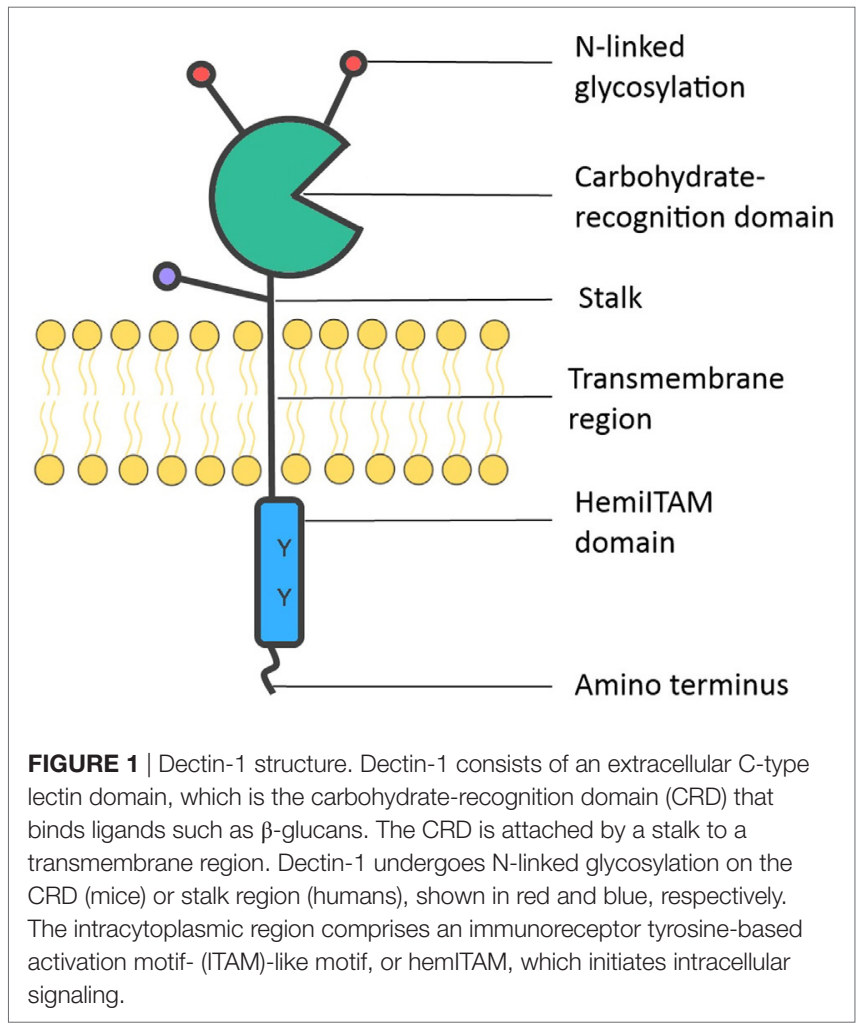




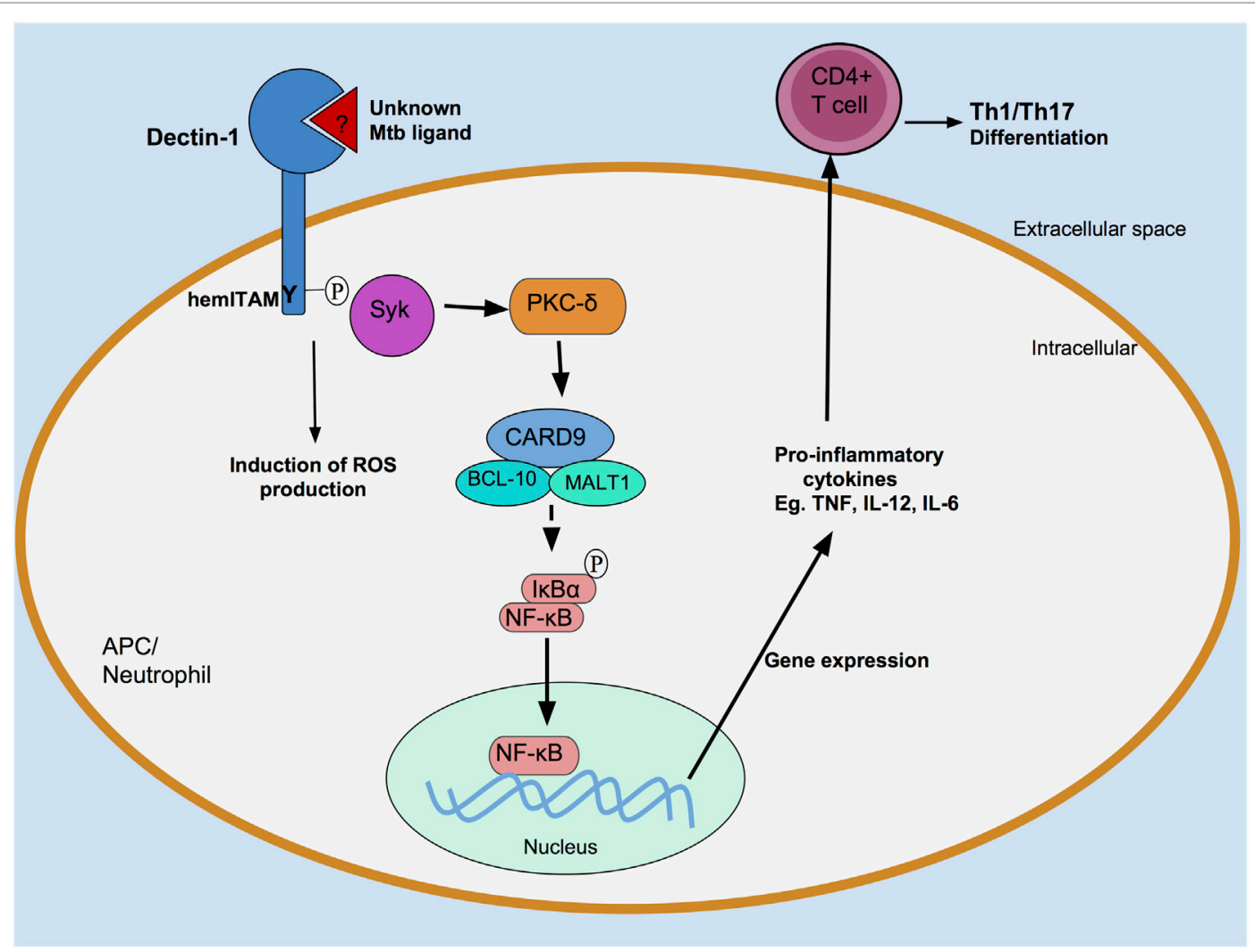

FIGURE 2 | Recognition of Mycobacterium tuberculosis (Mtb) by Dectin-1. An unknown Mtb ligand is recognized by Dectin-1, which is then tyrosine phosphorylated at its hemITAM residue by Src kinases (data not shown). This creates a docking site for spleen tyrosine kinase (Syk). Syk associates with a caspase recruitment domain family member 9 (CARD9)/B-cell lymphoma 10 (BCL-10)/mucosa-associated lymphoid tissue lymphoma translocation protein 1 (MALT1) protein complex, resulting in activation of the transcription factor nuclear factor $\mathrm{kB}$ (NF-kB). Pro-inflammatory cytokine production follows, which induce an adaptive T cell response. Dectin-1-dependent signaling can also occur in neutrophils, where they induce reactive oxygen species (ROS) production.

Dectin-1 does not appear to initiate protective responses in isolation, but acts synergistically with other receptors such as the TLRs. For instance, Dectin-1 stimulation was found to augment TLR-2-mediated production of cytokines in murine macrophages and DCs $(30,31)$. In addition, Ferwerda et al. showed that stimulation of human peripheral blood mononuclear cells (PBMCs) with a Dectin-1 ligand, as well as ligands for TLR-2 or -4, led to a synergistic increase in TNF- $\alpha$ production compared with Dectin-1 stimulation alone (37). Shin et al. extended this investigation to mycobacteria by infecting murine macrophages with M. abscessus (Mab), an environmental non-tuberculous Mycobacterium that can cause opportunistic infections in humans (38). The authors observed that Mab stimulation of macrophages initiated a physical colocalization between Dectin-1 and TLR-2 that was required for pro-inflammatory cytokine production (38). The mechanism underlying this apparent interaction remains to be elucidated $(23,39)$. In contrast to these findings, a study by Rothfuchs et al. showed that Dectin-1 inhibition significantly diminished the production of IL-12p40 by DCs lacking TLR-2 (40). This suggests that Dectin-1 signaling is not necessarily dependent on TLR-2. Notably, these conflicting studies described the effect of different microorganisms and stimuli on different cell types.

\section{DECTIN-1 RECOGNITION OF MYCOBACTERIA}

Dectin- 1 has been well characterized as a major fungal $\beta$-glucan receptor (41). Interestingly, Dectin-1 has also been shown to be involved in the innate immune recognition of mycobacteria (26), which do not contain $\beta$-glucans (40). Yadav and Schorey established this by infecting murine bone marrow-derived macrophages (BMDMs) with non-pathogenic M. smegmatis. They found that the production of TNF- $\alpha$ by BMDMs was decreased by approximately $60 \%$ in the presence of Dectin-1-blocking antibodies (26). Moreover, they showed that Dectin-1 was required for the production of IL-6, G-CSF, and RANTES by BMDMs (26). However, when the authors performed infection using more virulent mycobacterial strains, such as Mtb strain H37Rv, they observed a significant decrease in the production of TNF- $\alpha$ compared with non-pathogenic strains, and this minimal TNF$\alpha$ production was not reliant on Dectin-1 (26). This suggested that the role of Dectin-1 in orchestrating immune responses to pathogenic mycobacteria is somewhat limited. In contrast to this finding, a later study by Rothfuchs et al. reported that Dectin-1 does interact with pathogenic mycobacteria (40). These authors 
demonstrated that murine splenic DCs (spDCs) infected with pathogenic Mtb produced significantly less IL-12p40 when treated with laminarin, a competitive inhibitor of Dectin-1 (40). Thus, Dectin-1 is involved in the recognition of the important human pathogen Mtb as well as less virulent mycobacteria. Rothfuchs et al. went on to show that pharmacological Syk inhibition reduced the capacity of spDCs to produce IL-12p40 upon Mtb exposure, suggesting that this response was Syk dependent (40). The authors also elegantly confirmed the presence of a Dectin-1 ligand on mycobacteria, by showing that a Dectin-1-Fc fusion protein (42) was capable of binding live M. bovis (40). These and other related studies established the role of Dectin-1-mediated responses in non-human innate immune cells. The role of this CLR in human cells was investigated more recently.

Although they are not traditionally considered to be immune cells, airway epithelial cells may serve an important function in mediating pulmonary immune responses. These cells express PRRs such as Dectin-1, albeit to a lesser extent than myeloid cells. Lee et al. reported that Mtb could induce Dectin-1 expression in human A549 airway epithelial cells in a TLR-2-dependent fashion (43), and such expression contributed to the production of reactive oxygen species (ROS), antimicrobial peptides, and pro-inflammatory cytokines by these cells (43). Another investigation into mycobacteria-induced Dectin-1 signaling in human cells was undertaken by Zenaro et al. These authors observed that Mtb infection induced the maturation of monocyte-derived DCs (MDCs), as well as the Dectin-1-dependent production of IL-1 $\beta$, IL-6, IL-23, and TNF- $\alpha$ (44) by these cells. In addition, DCs activated with a Dectin-1 agonist stimulated naïve $\mathrm{CD}^{+}$ T cells to secrete IFN- $\gamma$ and IL-17 (44). Another study has demonstrated that simultaneous activation of neonatal MDCs with agonists of Dectin-1 and TLRs promote synergistic production of IL-12p70 (45). In human PBMCs stimulated with Mtb, Dectin-1 and TLR- 4 are the main receptors driving IL-17A production (46). Interestingly, administration of Dectin-1 agonist, curdlan, together with a mycobacterial antigen TB10.4, induced Th1 and Th17 responses in neonatal mice infected with Mtb (45). A recent study by Bisiaux et al. investigated cell-specific activation and proinflammatory responses in human whole blood stimulated with BCG (47). Interestingly, Dectin-1/2 responses were predominantly activated in neutrophils when compared with monocytes and lymphocytes populations. This work also demonstrated that induction of ROS production by BCG was decreased by neutralization of Dectin-1/2 and TLR-2/4 in both neutrophils and monocytes (47). In agreement with these findings, a recent report has shown that Mtb can induce DC maturation by generating ROS production through Dectin-1/TLR-2 (48). These in vitro findings indicated that Dectin-1 signaling is involved in the stimulation and activation of neutrophils and antigen-presenting cells (APCs), which lead to adaptive anti-mycobacterial immune responses.

To explore the significance of Dectin-1 in vivo, Marakalala et al. investigated Dectin-1-deficient mice and wild-type (WT) controls infected with aerosolized Mtb (49). The kncokout mice had significantly and reproducibly decreased $(\sim 0.5 \mathrm{log})$ pulmonary bacillary burdens (49). However, both the Dectin-1-deficient and WT mice developed similar histological signs of pneumonia, suggesting that there was still profound inflammatory activation in Dectin-1-deficient mice despite their decreased bacterial burden. Indeed, Dectin-1 deficiency did not result in any significant and reproducible changes in the pulmonary cytokine expression profiles compared with WT controls (49). The authors also found that Dectin-1 deficiency did not significantly affect mouse survival 150 days of post-infection. Marakalala et al. concluded that Dectin-1 does not play a major role in immunity to Mtb in vivo and acknowledged the potential significance of the mechanisms underlying the decreased bacillary burden conferred by Dectin-1 deficiency.

\section{THE FUNCTION OF Syk in PRR SIGNALING AND IMMUNITY TO Mtb}

Spleen tyrosine kinase is an intracellular signal transducer that performs diverse biological functions, including innate immune recognition (50). Syk can be activated by associating with the phosphorylated ITAM or hemITAM motif of CLRs via one of its two SH2 domains. In the case of the Dectin-1/Syk pathway, Syk activates protein kinase C-delta (PKC $)$ which mediates the phosphorylation of CARD9 (51). This enables CARD9 to associate with B-cell lymphoma 10 and the paracaspase mucosaassociated lymphoid tissue lymphoma translocation protein 1 (MALT1) to form a trimolecular structure capable of canonically activating NF- $\mathrm{KB}(52,53)$. Such manipulation of gene expression allows Syk to exert its secondary messenger functions, triggering ROS production (33), accelerated phagocytosis (28), and the production of pro-inflammatory cytokines, such as IL-1 $\beta$, IL-6, IL-12, and TNF- $\alpha$ (18). Mature IL- $1 \beta$ production requires further post-translational modification of pro-IL- $1 \beta$ by a caspase1-containing oligomer called an inflammasome (50). The NLPR3 inflammasome is activated in a Syk-dependent mechanism by mycobacteria that express the virulence factor ESAT6, and such activation has been linked to macrophage necrosis (54). There is therefore mounting evidence that this signaling molecule may be relevant in immune responses to mycobacterial infections.

Spleen tyrosine kinase is involved in a multitude of biological functions, and therefore essential for normal survival in mice (55). This makes it difficult to determine the effect of Syk deficiency on mycobacterial immunity in vivo, for example, using a gene knockout study in mice. Better research tools are required to understand the role of Syk in mycobacterial infections in vivo and in humans.

\section{PKCס AND IMMUNITY TO Mtb}

As already described, CLRs which activate Syk are linked to the CARD9-BCL10-MALT1 pathway. Strasser et al. showed that the linker molecule PKC $\delta$ is activated by Syk and mediates the phosphorylation of CARD9 (51). The role of this kinase in signal transduction prompted Parihar et al. to investigate the relevance of PKC $\delta$ function following Mtb infection (56). They observed that mice deficient in PKCS (either through gene knockout or pharmacological inhibition) were less resistant to Mtb, displaying more severe lung pathology, excessive pro-inflammatory 
cytokine production, increased bacterial burdens, and increased mortality compared with their WT or untreated controls. Using gene expression profiles from Mtb-infected humans, the authors showed that PKC $\delta$ abundance was temporally associated with progression to TB disease. In addition, the authors analyzed lung specimens from TB patients and found that PKC $\delta$ was highly expressed in necrotic and cavitary granulomas (56). These data indicate that PKC $\delta$ is an important determinant of Mtb infection in humans and mice.

\section{CARD9 AND IMMUNITY TO Mtb}

CARD9 is an adaptor protein involved in the Dectin-1/Syk signaling cascade and is expressed in DCs and macrophages (52). The adaptor picks up on signals from multiple receptor classes, such as ITAM-based receptors (including CLRs) and TLRs (27). The role of CARD9 in Mtb immunity was evaluated by Dorhoi et al. using a mouse model of Mtb H37Rv infection (57). These authors demonstrated that CARD9-deficient mice had a reduced ability to control bacterial replication, developed severe lung pathology, and displayed increased mortality compared with their littermate controls (57). Infected CARD9-deficient mice developed acute pneumonia, and histological examination of the lungs revealed the presence of necrotic foci and an inflammatory infiltrate with profound neutrophil accumulation. Moreover, lung specimens from CARD9-deficient mice showed increased apoptotic cell death and secondary necrosis compared with WT controls (57). There is evidence to suggest that the mobilization of neutrophils from the bone marrow to peripheral tissues is dependent on the cytokines G-CSF and CXCL1 (58), and these cytokines were significantly elevated in the sera of CARD9-deficient mice compared with their littermate controls (57). The investigators also demonstrated that neutrophils deficient in CARD9 were unable to produce the regulatory cytokine IL-10 when challenged with Mtb, leading to deregulated pulmonary inflammation (57). These data led the authors to conclude that CARD9 is required for properly regulated innate immune cell activation during Mtb infection.

To further explore the mechanism of CARD9 function in TB, Dorhoi et al. infected APCs with Mtb H37Rv and found that deficiency in the CARD9 adaptor did not affect NO synthesis by the APCs nor did it affect the phagocytosis or destruction of Mtb following IFN- $\gamma$ activation (57). However, CARD9-deficient BMDMs produced significantly less TNF- $\alpha$, IL- $1 \beta$, IL-6, IL-12, and CCL5 compared with WT controls (57). Given the reduced levels of IL-12 in their in vitro experiments, one might have expected to see impaired Th1 responses in vivo (as IL-12 contributes to Th-1 polarization). However, no such aberrations in $\mathrm{T}$ cell recruitment and activation were observed in the lungs of infected CARD9-deficient mice (57). Similarly, the authors did not observe any deficit in Th17 responses in CARD9-deficient mice (57), even though an earlier study demonstrated that the Syk/CARD9 pathway is required for developing Th17 responses to Candida albicans (34). Nevertheless, adequate T-cell responses were not able to overcome the pathology induced by defects in innate immune cell inflammatory activation. These results suggest an essential role for CARD9 in anti-mycobacterial immunity.

\section{THE Syk/CARD9 PATHWAY IN TB VACCINOLOGY}

To generate a protective immune response to $\mathrm{Mtb}$, antigen-specific Th1 cells are required (59). In addition, the generation of Th17 cells secretingIL-17has been shown toaugment a protectivehostresponse by stimulating the influx of effector cells to the areas of infection (60). An effective vaccine to Mtb would probably need to activate APCs in such a way that they "instruct" $\mathrm{T}$ cell differentiation to Th1 and Th17 phenotypes. One novel vaccination strategy involves using recombinant Mtb antigens as subunit vaccines; however, these vaccines have not been successful on their own, perhaps because in themselves they do not activate APCs. This necessitates the use of vaccine adjuvants capable of triggering helpful innate cellular responses to guide adaptive immunity. Potential adjuvants include the mycobacterial PAMPs TDM and its synthetic analog TDB, which induce protective Th 1 and Th17 immunity by activating APCs via the Syk-CARD9-BCL10-MALT1 pathway (11). The major PRRs that bind TDM are Mincle $(7,8)$ and Clecsf8 $(13,17)$, suggesting that signaling initiated by either one or both of these CLRs via the Syk/CARD9 pathway may be essential for protective immunity to Mtb.

\section{CONCLUSION AND FUTURE DIRECTIONS}

An important step toward a deeper understanding of Dectin-1Syk-CARD9 signaling will be identifying the mycobacterial PAMP recognized by Dectin-1. This would help elucidate how Mtb interacts with human innate immune cells, and how this contributes to or lessens pathology. $\beta$-Glucans are currently the only known Dectin-1 ligand, yet Dectin-1 recognizes mycobacteria, which do not express $\beta$-glucans. The nature of the ligand will therefore broaden our knowledge of Dectin-1 PAMP recognition.

Dectin-1 itself may not be essential for Mtb immunity, but some of the downstream signaling molecules such as CARD9 evidently are. The effect of other signaling molecules, like Syk and $\mathrm{PKC} \delta$, in Mtb responses remains to be fully elucidated. In addition, future studies should look at how CLRs that utilize this signaling pathway interact, perhaps by performing gene knockouts of multiple receptors in mice. Although Dectin-1 appears to play a minor role in in vivo Mtb immunity, components of Dectin-1/Syk signaling can induce protective downstream host responses, and this pathway remains a potential target for vaccine adjuvants.

\section{AUTHOR CONTRIBUTIONS}

MW wrote the manuscript. All authors planned the manuscript content, analyzed the literature, wrote parts of, and edited the manuscript.

\section{FUNDING}

The authors acknowledge funding from the Wellcome Trust (MM, 206751/Z/17/Z), South African Medical Research Council (SAMRC) self-initiated research grant (http://www. 
mrc.ac.za) (MJM), The National Research Foundation (http:// www.nrf.ac.za) (MM and JH), SAMRC with funding from South African Department of Health (http://www.mrc.ac.za), and the

\section{REFERENCES}

1. Medzhitov R, Janeway CA. Decoding the patterns of self and nonself by the innate immune system. Science (2002) 296:298-300. doi:10.1126/ science. 1068883

2. Medzhitov R. Recognition of microorganisms and activation of the immune response. Nature (2007) 449:819-26. doi:10.1038/nature06246

3. Marakalala MJ, Ndlovu H. Signaling C-type lectin receptors in antimycobacterial immunity. PLoS Pathog (2017) 13:e1006333. doi:10.1371/journal. ppat. 1006333

4. Ishikawa E, Mori D, Yamasaki S. Recognition of mycobacterial lipids by immune receptors. Trends Immunol (2017) 38:66-76. doi:10.1016/j.it.2016. 10.009

5. Yonekawa A, Saijo S, Hoshino Y, Miyake Y, Ishikawa E, Suzukawa M, et al. Dectin-2 is a direct receptor for mannose-capped lipoarabinomannan of mycobacteria. Immunity (2014) 41:402-13. doi:10.1016/j.immuni.2014.08.005

6. Kerscher B, Willment JA, Brown GD. The Dectin-2 family of C-type lectin-like receptors: an update. Int Immunol (2013) 25:271-7. doi:10.1093/intimm/ dxt006

7. Ishikawa E, Ishikawa T, Morita YS, Toyonaga K, Yamada H, Takeuchi O, et al. Direct recognition of the mycobacterial glycolipid, trehalose dimycolate, by C-type lectin Mincle. J Exp Med (2009) 206:2879-88. doi:10.1084/ jem.20091750

8. Schoenen $\mathrm{H}$, Bodendorfer $\mathrm{B}$, Hitchens $\mathrm{K}$, Manzanero S, Werninghaus $\mathrm{K}$, Nimmerjahn F, et al. Cutting edge: Mincle is essential for recognition and adjuvanticity of the mycobacterial cord factor and its synthetic analog trehalose-dibehenate. J Immunol (2010) 184:2756-60. doi:10.4049/jimmunol. 0904013

9. Lang R. Recognition of the mycobacterial cord factor by Mincle: relevance for granuloma formation and resistance to tuberculosis. Front Immunol (2013) 4:5. doi:10.3389/fimmu.2013.00005

10. Miyake Y, Ishikawa E, Ishikawa T, Yamasaki S. Self and nonself recognition through C-type lectin receptor, Mincle. Self Nonself (2010) 1:310-3. doi:10.4161/self.1.4.13736

11. Werninghaus K, Babiak A, Groß O, Hölscher C, Dietrich H, Agger EM, et al. Adjuvanticity of a synthetic cord factor analogue for subunit Mycobacterium tuberculosis vaccination requires FcR $\gamma$-Syk-Card9-dependent innate immune activation. J Exp Med (2009) 206:89-97. doi:10.1084/jem.20081445

12. Heitmann L, Schoenen H, Ehlers S, Lang R, Hölscher C. Mincle is not essential for controlling Mycobacterium tuberculosis infection. Immunobiology (2013) 218:506-16. doi:10.1016/j.imbio.2012.06.005

13. Miyake Y, Toyonaga K, Mori D, Kakuta S, Hoshino Y, Oyamada A, et al. C-type lectin MCL is an FcR $\gamma$-coupled receptor that mediates the adjuvanticity of mycobacterial cord factor. Immunity (2013) 38:1050-62. doi:10.1016/j. immuni.2013.03.010

14. Miyake Y, Oh-hora M, Yamasaki S. C-type lectin receptor MCL facilitates mincle expression and signaling through complex formation. JImmunol (2015) 194:5366-74. doi:10.4049/jimmunol.1402429

15. Graham LM, Gupta V, Schafer G, Reid DM, Kimberg M, Dennehy KM, et al. The C-type lectin receptor CLECSF8 (CLEC4D) is expressed by myeloid cells and triggers cellular activation through syk kinase. J Biol Chem (2012) 287:25964-74. doi:10.1074/jbc.M112.384164

16. Zhao XQ, Zhu LL, Chang Q, Jiang C, You Y, Luo T, et al. C-type lectin receptor Dectin-3 mediates trehalose 6,6'-dimycolate (TDM)-induced Mincle expression through CARD9/Bcl10/MALT1-dependent nuclear factor (NF)- $\kappa B$ activation. J Biol Chem (2014) 289:30052-62. doi:10.1074/jbc.M114.588574

17. Wilson GJ, Marakalala MJ, Hoving JC, Van Laarhoven A, Drummond RA, Kerscher B, et al. The C-type lectin receptor CLECSF8/CLEC4D is a key component of anti-mycobacterial immunity. Cell Host Microbe (2015) 17:252-9. doi:10.1016/j.chom.2015.01.004
University of Cape Town (HN, JH, and MM). JCH is supported by a Carnegie Corporation DEAL fellowship and the National Research Foundation of South Africa.
18. Brown GD, Marakalala MJ, Graham LM. The role of Syk/CARD9-coupled C-type lectin receptors in immunity to Mycobacterium tuberculosis infections. Clin Dev Immunol (2010) 2010:567571. doi:10.1155/2010/567571

19. Toyonaga K, Torigoe S, Motomura Y, Kamichi T, Hayashi JM, Morita YS, et al. C-type lectin receptor DCAR recognizes mycobacterial phosphatidyl-inositol mannosides to promote a Th1 response during infection. Immunity (2016) 45:1245-57. doi:10.1016/j.immuni.2016.10.012

20. Tanne A, Ma B, Boudou F, Tailleux L, Botella H, Badell E, et al. A murine DC-SIGN homologue contributes to early host defense against Mycobacterium tuberculosis. J Exp Med (2009) 206:2205-20. doi:10.1084/jem. 20090188

21. Ariizumi K, Shen GL, Shikano S, Xu S, Ritter R, Kumamoto T, et al. Identification of a novel, dendritic cell-associated molecule, dectin-1, by subtractive cDNA cloning. J Biol Chem (2000) 275:20157-67. doi:10.1074/ jbc.M909512199

22. Taylor PR, Brown GD, Reid DM, Willment JA, Martinez-Pomares L, Gordon S, et al. The beta-glucan receptor, dectin-1, is predominantly expressed on the surface of cells of the monocyte/macrophage and neutrophil lineages. J Immunol (2002) 169:3876-82. doi:10.4049/jimmunol.169.7.3876

23. Brown GD. Dectin-1: a signalling non-TLR pattern-recognition receptor. Nat Rev Immunol (2006) 6:33-43. doi:10.1038/nri1745

24. Reid DM, Montoya M, Taylor PR, Borrow P, Gordon S, Brown GD, et al. Expression of the beta-glucan receptor, Dectin-1, on murine leukocytes in situ correlates with its function in pathogen recognition and reveals potential roles in leukocyte interactions. J Leukoc Biol (2004) 76:86-94. doi:10.1189/ jlb.0104031

25. Brown GD, Gordon S. Immune recognition. A new receptor for beta-glucans. Nature (2001) 413:36-7. doi:10.1038/35092620

26. Yadav M, Schorey JS. The $\beta$-glucan receptor dectin-1 functions together with TLR2 to mediate macrophage activation by mycobacteria. Blood (2006) 108:3168-75. doi:10.1182/blood-2006-05-024406

27. Hara H, Ishihara C, Takeuchi A, Imanishi T, Xue L, Morris SW, et al. The adaptor protein CARD9 is essential for the activation of myeloid cells through ITAM-associated and Toll-like receptors. Nat Immunol (2007) 8:619-29. doi:10.1038/ni1466

28. Rogers NC, Slack EC, Edwards AD, Nolte MA, Schulz O, Schweighoffer E, et al. Syk-dependent cytokine induction by dectin-1 reveals a novel pattern recognition pathway for C type lectins. Immunity (2005) 22:507-17. doi:10.1016/j.immuni.2005.03.004

29. Gringhuis SI, den Dunnen J, Litjens M, van der Vlist M, Wevers B, Bruijns SCM, et al. Dectin-1 directs T helper cell differentiation by controlling noncannonical NK-kB activation through Raf-1 and Syk. Nat Immunol (2009) 10:203-13. doi:10.1038/ni.1692

30. Gantner BN, Simmons RM, Canavera SJ, Akira S, Underhill DM. Collaborative induction of inflammatory responses by dectin-1 and toll-like receptor 2 . J Exp Med (2003) 197:1107-17. doi:10.1084/jem.20021787

31. Brown GD, Herre J, Williams DL, Willment JA, Marshall ASJ, Gordon S. Dectin-1 mediates the biological effects of $\beta$-glucans. J Exp Med (2003) 197:1119-24. doi:10.1084/jem.20021890

32. Herre J, Marshall ASJ, Caron E, Edwards AD, Williams DL, Schweighoffer E, et al. Dectin-1 uses novel mechanisms for yeast phagocytosis in macrophages. Blood (2004) 104:4038-45. doi:10.1182/blood-2004-03-1140

33. Underhill DM, Rossnagle E, Lowell CA, Simmons RM. Dectin-1 activates Syk tyrosine kinase in a dynamic subset of macrophages for reactive oxygen production. Blood (2005) 106:2543-50. doi:10.1182/blood-2005-031239

34. Leibund Gut-Landmann S, Gross O, Robinson MJ, Osorio F, Slack EC, Tsoni S, et al. Syk- and CARD9-dependent coupling of innate immunity to the induction of T helper cells that produce interleukin 17. Nat Immunol (2007) 8:630-8. doi:10.1038/ni1460 
35. LeibundGut-Landmann S, Osorio F, Brown GD, Sousa CRE. Stimulation of dendritic cells via the dectin-1/Syk pathway allows priming of cytotoxic T-cell responses. Blood (2008) 112:4971-80. doi:10.1182/blood-2008-05-158469

36. Geijtenbeek TBH, Gringhuis SI. C-type lectin receptors in the control of T helper cell differentiation. Nat Rev Immunol (2016) 16:433-48. doi:10.1038/ nri.2016.55

37. Ferwerda G, Meyer-Wentrup F, Kullberg BJ, Netea MG, Adema GJ. Dectin-1 synergizes with TLR2 and TLR4 for cytokine production in human primary monocytes and macrophages. Cell Microbiol (2008) 10:2058-66. doi:10.1111/j.1462-5822.2008.01188.x

38. Shin DM, Yang CS, Yuk JM, Lee JY, Kim KH, Shin SJ, et al. Mycobacterium abscessus activates the macrophage innate immune response via a physical and functional interaction between TLR2 and dectin-1. Cell Microbiol (2008) 10:1608-21. doi:10.1111/j.1462-5822.2008.01151.x

39. Arbibe L, Mira JP, Teusch N, Kline L, Guha M, Mackman N, et al. Toll-like receptor 2-mediated NF-kappa B activation requires a Rac1-dependent pathway. Nat Immunol (2000) 1:533-40. doi:10.1038/82797

40. Rothfuchs AG, Bafica A, Feng CG, Egen JG, Williams DL, Brown GD, et al. Dectin-1 interaction with Mycobacterium tuberculosis leads to enhanced IL-12p40 production by splenic dendritic cells. JImmunol (2007) 179:3463-71. doi:10.4049/jimmunol.179.6.3463

41. Plato A, Hardison SE, Brown GD. Pattern recognition receptors in antifungal immunity. Semin Immunopathol (2015) 37:97-106. doi:10.1007/ s00281-014-0462-4

42. Graham LM, Tsoni SV, Willment JA, Williams DL, Taylor PR, Gordon S, et al. Soluble dectin-1 as a tool to detect $\beta$-glucans. J Immunol Methods (2006) 314:164-9. doi:10.1016/j.jim.2006.05.013

43. Lee HM, Yuk JM, Shin DM, Jo EK. Dectin-1 is inducible and plays an essential role for mycobacteria-induced innate immune responses in airway epithelial cells. J Clin Immunol (2009) 29:795-805. doi:10.1007/s10875-009-9319-3

44. Zenaro E, Donini M, Dusi S. Induction of Th1/Th17 immune response by Mycobacterium tuberculosis: role of dectin-1, mannose receptor, and DC-SIGN. J Leukoc Biol (2009) 86:1393-401. doi:10.1189/jlb.0409242

45. Lemoine S, Jaron B, Tabka S, Ettreiki C, Deriaud E, Zhivaki D, et al. Dectin-1 activation unlocks IL12A expression and reveals the TH1 potency of neonatal dendritic cells. J Allergy Clin Immunol (2015) 136:1355.e-68.e. doi:10.1016/j. jaci.2015.02.030

46. van de Veerdonk FL, Teirlinck AC, Kleinnijenhuis J, Kullberg BJ, van Crevel R, van der Meer JWM, et al. Mycobacterium tuberculosis induces IL-17A responses through TLR4 and dectin-1 and is critically dependent on endogenous IL-1. J Leukoc Biol (2010) 88:227-32. doi:10.1189/jlb.0809550

47. Bisiaux A, Boussier J, Duffy D, Quintana-Murci L, Fontes M, Albert ML. Deconvolution of the response to bacillus Calmette-Guérin reveals NF- $\mathrm{BB}$ induced cytokines as autocrine mediators of innate immunity. Front Immunol (2017) 8:796. doi:10.3389/fimmu.2017.00796

48. Romero MM, Basile JI, Corra Feo L, López B, Ritacco V, Alemán M. Reactive oxygen species production by human dendritic cells involves TLR2 and dectin-1 and is essential for efficient immune response against mycobacteria. Cell Microbiol (2016) 18:875-86. doi:10.1111/cmi.12562

49. Marakalala MJ, Guler R, Matika L, Murray G, Jacobs M, Brombacher F, et al. The Syk/CARD9-coupled receptor Dectin-1 is not required for host resistance to Mycobacterium tuberculosis in mice. Microbes Infect (2011) 13:198-201. doi:10.1016/j.micinf.2010.10.013

50. Mócsai A, Ruland J, Tybulewicz VLJ. The SYK tyrosine kinase: a crucial player in diverse biological functions. Nat Rev Immunol (2010) 10:387-402. doi:10.1038/nri2765

51. Strasser D, Neumann K, Bergmann H, Marakalala MJ, Guler R, Rojowska A, et al. Syk kinase-coupled C-type lectin receptors engage protein kinase $\mathrm{C}-\sigma$ to elicit Card9 adaptor-mediated innate immunity. Immunity (2012) 36:32-42. doi:10.1016/j.immuni.2011.11.015

52. Ruland J. CARD9 signaling in the innate immune response. Ann N Y Acad Sci (2008) 1143:35-44. doi:10.1196/annals.1443.024

53. Bertin J, Guo Y, Wang L, Srinivasula SM, Jacobson MD, Poyet JL, et al. CARD9 is a novel caspase recruitment domain-containing protein that interacts with BCL10/CLAP and activates NF-кB. J Biol Chem (2000) 275:41082-6. doi:10.1074/jbc.C000726200

54. Wong KW, Jacobs WR. Critical role for NLRP3 in necrotic death triggered by Mycobacterium tuberculosis. Cell Microbiol (2011) 13:1371-84. doi:10.1111/j.1462-5822.2011.01625.x

55. Turner M, Mee PJ, Costello PS, Williams O, Price AA, Duddy LP, et al. Perinatal lethality and blocked B-cell development in mice lacking the tyrosine kinase Syk. Nature (1995) 378:298-302. doi:10.1038/378298a0

56. Parihar SP, Ozturk M, Marakalala MJ, Loots DT, Hurdayal R, Beukes D, et al. Protein kinase C-delta (PKCס), a marker of inflammation and tuberculosis disease progression in humans, is important for optimal macrophage killing effector functions and survival in mice. Mucosal Immunol (2017). doi:10.1038/ mi. 2017.68

57. Dorhoi A, Desel C, Yeremeev V, Pradl L, Brinkmann V, Mollenkopf H-J, et al. The adaptor molecule CARD9 is essential for tuberculosis control. J Exp Med (2010) 207:777-92. doi:10.1084/jem.20090067

58. Wengner AM, Pitchford SC, Furze RC, Rankin SM. The coordinated action of G-CSF and ELR + CXC chemokines in neutrophil mobilization during acute inflammation. Blood (2008) 111:42-9. doi:10.1182/blood-2007-07-099648

59. Flynn JL, Chan J, Triebold KJ, Dalton DK, Stewart TA, Bloom BR. An essential role for interferon gamma in resistance to Mycobacterium tuberculosis infection. J Exp Med (1993) 178:2249-54. doi:10.1084/jem.178.6.2249

60. Khader SA, Bell GK, Pearl JE, Fountain JJ, Rangel-Moreno J, Cilley GE, et al. IL-23 and IL-17 in the establishment of protective pulmonary CD4+T cell responses after vaccination and during Mycobacterium tuberculosis challenge. Nat Immunol (2007) 8:369-77. doi:10.1038/ni1449

Conflict of Interest Statement: The authors declare that the research was conducted in the absence of any commercial or financial relationships that could be construed as a potential conflict of interest.

Copyright (C) 2018 Wagener, Hoving, Ndlovu and Marakalala. This is an open-access article distributed under the terms of the Creative Commons Attribution License (CC $B Y)$. The use, distribution or reproduction in other forums is permitted, provided the original author(s) and the copyright owner are credited and that the original publication in this journal is cited, in accordance with accepted academic practice. No use, distribution or reproduction is permitted which does not comply with these terms. 